\title{
A Simultaneous Descending Bid Auction for Multiple Items and Unitary Demand*
}

\author{
Marilda Sotomayor ${ }^{* *}$
}

Summary: 1. Introduction; 2. The competitive market and the auction mechanism; 3. The main results; 4. Concluding remarks.

Keywords: auction mechanism; competitive equilibrium; demand set.

JEL codes: C78 and D78.

This paper proposes a new descending bid method for auctioning multiple objects. The auctioneer announces a current price, bidders indicate their demands at that price, and the auctioneer lowers the prices until every object can be allocated to a buyer who demands it and no buyer receives more than one item. The features of this mechanism lie on the facts that: the objects are sold simultaneously at prices that clear the market; every two similar objects are sold at the same price; the mechanism always produces the same final price, namely, the maximum competitive equilibrium price. In addition, the auctioneer can choose an efficient allocation.

Este artigo propõe um novo método de lances descendentes para leiloar múltiplos objetos com demanda unitária. O leiloeiro anuncia um preço, os compradores indicam suas demandas ao preço anunciado, e o leiloeiro desce os preços até que todo objeto esteja alocado para algum comprador que o tenha demandado e ninguém esteja comprando mais do que um item. Os aspectos importantes deste mecanismo são que os objetos são vendidos simultaneamente a preços competitivos, quaisquer dois objetos similares são vendidos ao mesmo preço e independentemente das escolhas do leiloeiro o mecanismo sempre produz o mesmo preço final, o preço máximo de equilíbrio competitivo. Além disso, é sempre possível para o leiloeiro escolher uma alocação eficiente.

\footnotetext{
${ }^{*}$ This paper was received in nov. 2000 and approved in apr. 2001, having been partially supported by the Conselho Nacional de Desenvolvimento Científico e Tecnológico (National Council for Scientific and Technological Development) and Fundação Instituto de Pesquisas Econômicas (Economic Research Foundation), Brazil.

${ }^{* *}$ Economics Department/USP
} 


\section{Introduction}

Demange (1982), Leonard (1983), Demange and David (1985) and Sotomayor (1999) have considered an allocation mechanism that turns out to be a multiitem generalization of the "first price" auction described by Vickrey (1961). In this auction, the buyers submit sealed bids for a single item, which is sold to the highest bidder at a price equal to her bid. We can think of the first price pattern as an ordinary competitive equilibrium. At the selected price, the object is demanded by only one "consumer", the highest bidder, and, as there is only one item, this produces balance of supply and demand. The important property of the first highest bid is that it is the maximum competitive equilibrium price, since no one would demand the item for any higher price.

In the multi-item generalization, bidders want exactly one item of a set of indivisible objects. Each buyer attributes a monetary value to each of the items. In these models, a buyer has no additonal utility for any supplementary object, although she has preferences defined over the set of all objects. One may also think of the quota as an institutional restriction of the market, as is often the case in the public sector auctions, in which the restriction aims at limiting the market power of the winners. ${ }^{1}$ Examples of such a market are the real estate market ${ }^{2}$ and the market for used cars. ${ }^{3}$

Given a price for each object, a bidder will demand the object or objects that maximize her surplus, which is the difference between her valuation and the price of the object, assuming this surplus is non-negative. A price vector together with an allocation of the objects is a competitive equilibrium if every buyer can be assigned an item in her demand set and no two bidders are assigned the same object. In addition, if an item is unsold, then its price must equal its reservation price to the seller. This last condition is required for equilibrium, because if the price of an item exceeds its reservation price to the seller, there will be excess supply and the seller of the item will want to lower its price.

Competitive equilibria always exist. ${ }^{4}$ An interesting property of the set of

\footnotetext{
${ }^{1}$ In Brazil, the licenses to use cellular telephone services vary both in geographic coverage and amount of spectrum covered, and are classified in two types. Each type covers a geographic area corresponding to a certain social level of the population. These licenses were auctioned under the restriction that the buyers could buy two licenses, at most, one of each type. One can think of it as two markets of non-identical objects each, such that a buyer can buy one license, at most, in each market.

${ }^{2}$ When houses are bought to live in rather than as an investment, i.e., each buyer wants to buy one house and has preferences over different types of houses.

${ }^{3}$ When each buyer wants to buy exactly one car and has preferences over different features.

${ }^{4}$ Gale (1960) and Shapley and Shubik (1972) proved the existence of competitive equilibria by
} 
competitive equilibria is that, among the equilibrium price vectors, there is a unique one, $p$, which is bigger in the strong sense that $p$ is at least as big in every component as any other competitive price vector. Therefore, $p$ is the "best" competitive equilibrium price vector from the point of view of the sellers and it is called the maximum competitive equlibrium price. There is also a minimum competitive equilibrium price with symmetrical properties. ${ }^{5}$.

The multi-item auction mechanism requires each bidder to submit a sealed bid listing her valuation of all the items. The auctioneer then allocates the objects in accordance with the maximum competitive equilibrium price.

The purpose of this paper is to show that there is another property of the single item auction that generalizes to the multi-item case. Namely, instead of a one-shot sealed bid auction, it is possible to reach the maximum competitive equilibrium price allocation by a "dynamic" auction. This is a natural generalization of the familiar Dutch auction, in which the auctioneer begins by asking for a certain price of an item and systematically lowers it until some bidder claims the item. The auction then stops and the buyer pays the price indicated.

The main features of this mechanism lie on the facts that the objects are sold simultaneously at prices that clear the market, every two similar objects are sold for the same price, ${ }^{6}$ no buyer receives more than one object and the objects are allocated efficiently.

Demange et al. (1986) propose an ascending bid auction mechanism a version of the Hungarian algorithm for the assignment problem (Dantzig, 1963) and generalizes the Vickerey second-price auction. This auction, as well as Vickrey's, produces the minimum equilibrium price. Both mechanisms are non-manipulable by any set of buyers. ${ }^{7}$ Unfortunately, as we demonstrate here, this property does

using linear programming. There is also a simple proof of Sotomayor (1999) using combinatorial arguments. Demange et al. (1986) present two dynamic mechanisms that lead to the minimum competitive equilibria. Crawford and Knoer (1981) introduced one of these mechanisms, which is a generalization of the deferred acceptance algorithm of Gale and Shapley (1962). Quinze (1984),Kaneko (1982), Kaneko and Yoshitsugu (1986), Alkan (1988), Alkan (1992) and Alkan and Gale (1988) prove the existence of competitive equilibria in a more general model.

${ }^{5}$ Shapley and Shubik (1972) also proved the existence of the minimum and the maximum competitive equilibrium price. (Roth and Sotomayor, 1990)

${ }^{6}$ In 1981, Sotherby Parke Bernet ran a sequential auction to sell seven identical licenses to use RCA's communications satellite for cable television broadcasts. The winning bids varied widely. The highest was US\$ 14.4 million and the lowest was US\$10.7 million. The FCC nullified the auction saying the procedure was "unjustly discriminatory" in charging different prices for the same service and ordered RCA to charge the same price to all. This story is told in PR Newswere (Nov. 9, 1981), Cristian Science Monitor (June 29, 1982), and Time (Dec. 13, 1982, p. 148).

${ }^{7}$ This result was proved by Demange and David (1985) for a more general model. It is a particular case of theorem 9.23 of Roth and Sotomayor (1990), due to Sotomayor (1986). Vickrey 
not hold for the mechanism presented in this paper.

This paper is organized as follows. In section 2, we describe the competitive market and the auction mechanism. Section 3 is devoted to the main results. Section 4 concludes the paper.

\section{The Competitive Market and the Auction Mechanism}

There is a set $B$ with $m$ bidders and a set $Q$ with $n$ indivisible items. Each object $q$ has a reservation price of $c_{q}$. The value of object $q$ to bidder $b$ is $a_{b q} \geq 0$. For technical convenience, we will assume that $B$ contains a dummy bidder $b_{o}$, who values any object $q$ in $a_{o q}=c_{q}$. We will also assume that $Q$ contains a dummy object $q_{o}$, whose value $a_{b o}$ is zero for all bidders and whose price is always zero. In particular, $a_{o o}=0$. We will assume that all prices and valuations are integers. The competitive market is then given by $(B, Q, c, a)$, where $c$ is the vector of reservation prices and $a$ is the $m \times n$ matrix of values $a_{b q^{\prime} s}$.

A feasible price vector $p$, which will be called simply price vector, is a function from $Q$ to $R^{+}$, such that $p(q) \equiv p_{q}$ is greater than or equal to $c_{q}$. A matching $\mu$ is an allocation of the objects to the buyers that does not violate the quotas of the buyers. If $b$ and $q$ are matched under $\mu$ at prices $p$, the payoff of $b$ is $a_{b q}-p_{q}$ and we write $\mu(q)=b$ or $q=\mu(b)$. If $b$ is matched to $q_{o}$, we will sometimes say that $b$ is unmatched. In this case, her payoff is zero. The dummy buyer can be matched to more than one object and the dummy object can be allocated to more than one buyer. The matching $\mu$ is feasible if each non-dummy object is assigned to exactly one bidder and no non-dummy bider is matched to more than one object. The matching $\mu$ is optimal if $\sum_{q \in Q} a_{\mu(q) q} \geq \sum_{q \in Q} a_{\mu^{\prime}(q) q}$, for all feasible $\mu^{\prime}$. Thus, an optimal matching maximizes the gains of $B \cup Q$ and, thereby, it is an efficient allocation of the objects. When all items are similar, an optimal matching allocates the objects to the bidders who value them most.

The demand set of bidder $b$ at prices $p$ is defined by

$$
D_{b}(p)=\left\{q \in Q ; a_{b q}-p_{q}=\max _{q^{\prime} \in Q}\left\{a_{b q^{\prime}}-p_{q^{\prime}}\right\}\right\}
$$

That is, $q \in D_{b}(p)$ if $q$ is one of $b^{\prime} s$ most preferred objects at price $p$. Observe that $D_{b}(p)$ is always non-empty, because there is always the possibility of demanding the dummy object.

(1961) proved that telling the truth is a dominant strategy for each buyer under the second-price auction. Demange (1982) and Leonard (1983) extended Vickrey's result to the case of multi-items and unitary demand. 
The demanders of object $q$ at price $p$ are defined by:

$$
B_{q}(p) \equiv\left\{b \in B ; q \in D_{b}(p)\right\}
$$

That is, $B_{q}(p)$ is the set of buyers who demand object $q$ at price $p$. Since the dummy bidder always demands the dummy object, $B_{q}(p) \neq \phi$ when $q=q_{o}$.

Now we can redefine a competitive equilibrium price. The pair $(p, \mu)$ is a competitive equilibrium, if $B_{q}(p) \neq \phi$ for every $q \in Q$, and each object $q$ (including the dummy object $q_{o}$ ) is matched to a buyer (including the dummy buyer $b_{o}$ ) in $B_{q}(p)$ and no non-dummy buyer is assigned more than one object. The matching $\mu$ is said to be competitive. Consequently, $a_{b q}-p_{q} \leq 0$ for every pair $(b, q)$ such that $b$ is matched to $q_{o}$. Thus, at a competitive equilibrium, not only is every nondummy object is allocated to a buyer who demands it, but no unmatched buyer has a positive payoff. Shapley and Shubik (1972) demonstrated that a matching is optimal if and only if it is competitive.

The competitive equilibrium $(p, \mu)$ is a maximum competitive equilibrium if $p_{q} \geq p_{q}^{\prime}$ for all objects $q$ and all equilibrium price vector $p^{\prime}$.

Definition 2.1 Let $p$ be a feasible price. Suppose that $B_{q}(p) \neq \phi \quad \forall q \in Q$. Let $S \subseteq Q$. Set $T \equiv \cup_{q \in S} B_{q}(p)$. Then, $S$ is underdemanded at prices $p$ if $b_{o} \notin T$ and $|T|<|S|$.

We will call $T$ the set of demanders of $S$.

Definition 2.2 $S$ is minimal underdemanded at prices $p$ if no proper subset of $S$ is an underdemanded set.

If a competitive matching exists for the price vector $p$, each object in every subset $S$ of $Q$ must be matched to a different buyer, so $B_{q}(p) \neq \phi$ for all objects $q \in S$, and there must be at least as many buyers in $\cup_{q \in S} B_{q}(p)$ as there are objects in $S$. Hall's theorem says that this necessary condition is also sufficient. That is, a competitive matching for the price vector $\mathrm{p}$ exists if and only if there is no underdemanded set at prices p (Hall, 1935), Gale and Shapley (1962), Dantzig (1963)).

In order to describe our auction mechanism, we will assume that there exists some price vector $M=\left(M_{1}, M_{2}, \ldots, M_{n}\right)$ big enough, such that everyone knows that $a_{b q} \leq M_{q}, \forall(b, q) \in B \times Q$. (The component corresponding to $q_{o}$ is zero). 
Step 1 The auctioneer announces the starting price vector $M=\left(M_{1}, M_{2}, \ldots, M_{n}\right)$. Each bidder bids by announcing her revealed demand set at price $M$. (recall that $D_{b}(p)$ is never empty). Then, if there still exists some object $q$, such that $B_{q}(M)=\phi$, the auctioneer lowers its price by one unit, keeping the other prices unchanged. The auctioneer repeats this procedure until every object gets at least one demander. This defines the price $p(1)$.

Step $\mathbf{t}+1$ At the beginning of this step, every object $q$ with price $p(t)>c_{q}$, is demanded by some non-dummy buyer and every object $q$ (including $q_{o}$ ), with price $p(t)=c_{q}$ is demanded by at least $b_{o}$. If it is possible to find a matching where each object $q$ is matched to a buyer in $B_{q}(t)$, and every non-dummy buyer is not assigned to more than one object, then the auction ends. Otherwise, Hall's theorem implies that there is some set of non-dummy buyers who are the demanders of some set of underdemanded objects at price $p(t)$. The auctioneer chooses a minimal underdemanded set $S$ and lowers the price of each object in the set by one unit. All other prices remain unchanged. This defines $p(t+1)$.

It is clear that the algorithm stops at some step $t$, because as soon as the price of an object becomes equal to its reservation price, it can be allocated to the dummy buyer.

Now, let us assume that bidders are quite precise in their responses to changing prices. That is, at each stage of the mechanism each buyer demands all objects that maximize her surplus at the current prices. This requires that bidders construct their bids thinking that their values are given by some matrix $\left\{a_{b q}\right\}$, which will be called matrix of the indirectly revealed values. Then, for example, if a bidder demands $s$ objects at some step $t$, and $k$ of these objects have their prices lowered in the next step, then the bidder must demand these $k$ objects at step $t+1$. She cannot demand other items and she cannot switch from one object to a different one. This is a consequence of the requirement that her valuations be integral numbers of units. For the same reason, if a bidder demands $s$ objects at some step $t$ and none of these objects have their prices lowered, then the bidder must demand these $k$ objects and possibly others, whose prices were decreased by one unit at step $t+1$. Thus, if a bidder has a positive payoff, then she does not demand $q_{o}$, so she will not be unmatched. It then follows that the final price and the final matching obtained by this algorithm are competitive for the competitive market $(B, Q, c, a)$. Thus, the final allocation is a competitive equilibrium, so the final matching is efficient. What is less clear is that this algorithm yields the same price, independent of the minimal underdemanded sets chosen by the auctioneer along the auction. This will be proved in the next section. Indeed, the final price is 
always the maximum equilibrium price vector, although this fact is not so obvious.

There may be several competitive matchings and so, there may be several possibilities of choice for the auctioneer at the last step of the auction. It is interesting, and by no means obvious, that the payoffs of the bidders do not depend on the competitive matching selected by the auctioneer. This is because every competitive price together with the corresponding payoff vector for the buyers is a core payoff, every competitive matching is optimal and every optimal matching is compatible with any core payoff. (Shapley and Shubik, 1972); (Roth and Sotomayor, 1990).

The example that follows illustrates the mechanism.

Example Let $B=\left\{b_{1}, \ldots, b_{4}, b_{o}\right\}, Q=\left\{1,2,3, q_{o}\right\}$ and $c=\{0,0,0,0\}$. The rows of the matrix of the indirectly revealed values are $(7,6,4,0),(6,4,3,0),(5,5,2$, $0)$ and $(3,6,4,0)$, respectively. These numbers define the matrix $\left(a_{b q}\right)_{b, q}$. Suppose that everyone knows that $M=(20,10,8,0)$ is such that $a_{b q} \leq M_{q} \forall(b, q) \in B \times Q$.

Step 1 The auctioneer announces the price vector $p(1)=(20,10,8,0)$ and the bidders demand $q_{o}$. Then he proceeds to decrease the three first components progressively by one unit each time. The first change in demands occurs when the price vector $(16,6,4,0)$ is reached. Bidders $b_{1}$ and $b_{4}$ indicate that they demand objects 2 and 3 ; bidder $b_{o}$ always demands $q_{o}$. No one demands object 1 . Then, the price of object 1 is lowered until the price vector $(7,6,4,0)$ is reached. At this price, $b_{1}$ demands all four objects and $b_{4}$ demands 2,3 and $q_{o}$. Since it is not possible to allocate each object to a buyer who demands it, so that no non-dummy buyer receives more than one object, the auctioneer finds a minimal underdemanded set. The minimal underdemanded set is $S_{1}=\{1,2,3\}$ and the set of demanders of $S_{1}$ is $T_{1}=\{b 1, b 4\}$. Then, the auctioneer lowers the prices of the three objects by one unit. This defines $p(2)$.

Step $2 p(2)=(6,5,3)$. Bidder $b_{1}$ demands 1,2 and 3 ; bidder $b_{2}$ demands 1,3 and $q_{o}$; bidder $b_{3}$ demands 2 and $q_{o}$; bidder $b_{4}$ demands 2 and 3 and the dummy bidder demands only $q_{o}$. There are three possible choices for the auctioneer: $\mu_{1}(1)=$ $b_{1}, \mu_{1}(2)=b_{3}, \mu_{1}(3)=b_{4}, \mu_{1}\left(q_{o}\right)=\left\{b_{2}, b_{o}\right\} ; \mu_{2}(1)=b_{1}, \mu_{2}(2)=b_{4}, \mu_{2}(3)=$ $b_{2}, \mu_{2}\left(q_{o}\right)=\left\{b_{3}, b_{o}\right\}$; and $\mu_{3}(1)=b_{2}, \mu_{3}(2)=b_{1}, \mu_{3}(3)=b_{4}, \mu_{3}\left(q_{o}\right)=\{b 3, b o\}$. All these matchings are competitive. The payoff vector of the bidders is $(1,0,0$, $1,0)$, under any of the three matchings. The final price is $(6,5,3)$, which is the maximum competitive price for the competitive market given. 


\section{The Main Results}

In this section, we demonstrate that the final price $p$ yielded by the auction mechanism is the maximum competitive price for the market $(B, Q, c, a)$, where $a$ is the matrix of the indirectly revealed values. Next, we show that similar objects are sold for the same price. Finally, we prove that the auction is manipulable by the buyers.

In the proof of theorem 1, we will use the following notation. Given a price $p, u_{b}(p) \equiv \max _{q \in Q} a_{b q}-p_{q}$. We will write $u_{b}(t)$ and $u_{b}(t+1)$ to indicate $u_{b}(p(t))$ and $u_{b}(p(t+1))$, respectively.

Theorem 3.1 Let $p$ be the price vector obtained from the auction mechanism. Then, $p$ is the maximum competitive price for the market $(B, Q, c, a)$.

Proof Suppose, by way of contradiciton, that there exists a competitive price $y$ such that $p \ngtr y$. At step $t=1$ of the auction, we have $p(1) \geq a_{b q}$, for all $(b, q) \in B \times Q$, so $p(1) \geq y$. Let $t$ be the last step of the auction at which $p(t) \geq y$. It is easy to see that

$$
u_{b}(t) \leq u_{b}(y) \text { for all } b \in B
$$

In fact, let $q \in D_{b}(t)$. Then, using that $p(t) \geq y$, we get that $u_{b}(t)=a_{b q}-p_{q}(t) \leq$ $a_{b q}-y_{q} \leq u_{b}(y)$.

Let $S$ be the minimal underdemanded set whose prices are decreased at step $t+1$. Thus, $S=\left\{q ; p_{q}(t+1)<p_{q}(t)\right\}$. Then, if $q \in S, p_{q}(t)>p_{q}(t+1) \geq c_{q}$, from which follows that $q \neq q_{o}$ and $q$ is not demanded by $b_{o}$ at prices $p(t)$. Let $T$ be the set of demanders of $S$ at $p(t)$, that is, $T=\cup_{q \in S} B_{q}(t)$. Then, $b_{o} \notin T$. We claim that $T=T^{\prime}$ where $T^{\prime}=\left\{b \in B ; u_{b}(t+1)>u_{b}(t)\right\}$. In fact, observe that if $q \in S$ and $b \in B_{q}(t)$, then $u_{b}(t)=a_{b q}-p_{q}(t)<a_{b q}-p_{q}(t+1) \leq u_{b}(t+1)$, so $b \in T^{\prime}$. Thus, $T \subseteq T^{\prime}$. Now, if $b \in T^{\prime}$, then there is $q \in S$ such that $b \in B_{q}(t)$, for suppose not. Then, $q \notin S$ for all $q \in D_{b}(t)$, so $p_{q}(t)=p_{q}(t+1)$ for all $q \in D_{b}(t)$. Let $q \in D_{b}(t+1)$. Therefore, $u_{b}(t)<u_{b}(t+1)=a_{b q}-p_{q}(t+1)$, so $u_{b}(t)<a_{b q}-p_{q}(t+1)$. If $q \in D_{b}(t)$, we get that $u_{b}(t)<a_{b q}-p_{q}(t)$, which contradicts the definition of $u_{b}(t)$. Then, $q \notin D_{b}(t)$, so $p_{q}(t)=p_{q}(t+1)+1$. On the other hand, $u_{b}(t)>a_{b q}-p_{q}(t)=a_{b q}-\left[p_{q}(t+1)+1\right]$, where in the inequality it was used that $q \notin D_{b}(t)$. Hence, $u_{b}(t) \geq a_{b q}-p_{q}(t+1)=u_{b}(t+1)$, which contradicts the fact that $b \in T^{\prime}$. 
That $S$ is underdemanded means exactly that

$$
|T|<|S|
$$

Now, define $T_{1}=\left\{b ; u_{b}(t+1)>u_{b}(y)\right\}$. That $T_{1} \neq \phi$, follows from the fact that there exists some $q$ such that $p_{q}(t+1)<y_{q}$, for $t$ is the last step at which $p(t) \geq y$. Thus, for $b \in B_{q}(y)$, we have $u_{b}(t+1) \geq a_{b q}-p_{q}(t+1)>a_{b q}-y_{q}=$ $u_{b}(y)$, so $b \in T_{1}$ and $T_{1} \neq \phi$. It is clear that $T_{1} \subseteq T$, because if $b \in T_{1}$, then $u_{b}(t+1)>u_{b}(y) \geq u_{b}(t)$, where the last inequality follows from (1). We also have that

$$
u_{b}(t)=u_{b}(y) \forall b \in T_{1}
$$

because $u_{b}(t+1)=u_{b}(t)+1$ (recall that all prices and valuations are integers), so $u_{b}(t)+1>u_{b}(y) \geq u_{b}(t)$, from which follows that $u_{b}(y)=u_{b}(t)$.

Define $S_{1}=\left\{q \in S ; B_{q}(t) \cap T_{1} \neq \phi\right\}$. We are going to show that

$$
\left|T_{1}\right| \geq\left|S_{1}\right|
$$

If this is done, then (2) and (4) give $0 \leq\left|T-T_{1}\right|<\left|S-S_{1}\right|$, so $S-S_{1} \neq \phi$. However, $S-S_{1}=\left\{q \in S ; B_{q}(t) \subseteq T-T_{1}\right\}$ (if $q \in S$, then $B_{q}(t) \subseteq T$ ) and $\cup_{S-S 1} B_{q}(t) \subseteq T-T_{1}$. Then, $\left|\cup_{S-S 1} B_{q}(t)\right| \leq\left|T-T_{1}\right|<\left|S-S_{1}\right|$. Since $b_{o} \notin T$, it implies that $b_{o} \notin \cup_{S-S 1} B_{q}(t)$. So, $S-S_{1}$ is underdemanded, which contradicts the minimality of $S$.

In order to show (4), we have to establish that $B_{q}(y) \subseteq T_{1}, \forall q \in S_{1}$. If this is done, then we will get that $\cup_{S_{1}} B_{q}(y) \subseteq T_{1}$. On the other hand, the competitiveness of $q$ implies that $S_{1}$ is not underdemanded, so, since $b_{o} \notin \cup_{S_{1}} B_{q}(y)$ (because $\left.b_{o} \notin T_{1}\right)$, we must have that $\left|\cup_{S_{1}} B_{q}(y)\right| \geq\left|S_{1}\right|$ and so $\left|T_{1}\right| \geq\left|S_{1}\right|$. Hence, take $q \in S_{1}$. Then, $B_{q}(t) \cap T_{1} \neq \phi$, so there is some $b \in B_{q}(t) \cap T_{1}$. If $k \notin T$, then since $q \in S_{1} \subseteq S$ and $T=\cup_{q \in S} B_{q}(t)$, we must have that $k \notin B_{q}(t)$. Then , $q \notin D_{k}(t)$. Thus, if $s \in D_{k}(t), a_{k s}-y_{s} \geq a_{k s}-p_{s}(t)>a_{k q}-p_{q}(t)=a_{k q}-\left(a_{b q}-\right.$ $\left.u_{b}(t)\right)=a_{k q}-\left(a_{b q}-u_{b}(y)\right)=a_{k q}-a_{b q}+u_{b}(y) \geq a_{k q}-a_{b q}+a_{b q}-p_{q}(y)$, where in the first inequality it was used that $p(t) \geq y$; in the second it was used that $s \in D_{k}(t)$ and $q \notin D_{k}(t)$; the first and second equalities used that $b \in B_{q}(t)$ and (3), respectively, and the third inequality used that $u_{b}(y) \geq a_{b q}-p_{q}(y)$. Therefore, $a_{k s}-y_{s}>a_{k q}-p_{q}(y)$. Then, $q \notin D_{k}(y)$, so $k \notin B_{q}(y)$.

If $k \in T-T_{1}$, then $u_{k}(t)<u_{k}(t+1) \leq u_{k}(y)$. Therefore, $a_{k q}-p_{q}(t) \leq$ $u_{k}(t)<u_{k}(y)$ and so, $u_{k}(y)>a_{k q}-p_{q}(t)$. Thus, $q \notin D_{k}(y)$, so $k \notin B_{q}(y)$. Hence, $B_{q}(y) \subseteq T_{1}$ and the proof is complete. 
Proposition 3.2 Let $p$ be some competitive price for $(B, Q, c, a)$. Let $q$ and $q *$ be such that $c_{q}=c_{q} *$ and $a_{b q}=a_{b q^{*}}$ for all $b \in B$. Then, $p_{q}=p_{q^{*}}$.

Proof Let $\mu$ be some competitive matching. Suppose, by way of contradiction, that $p_{q} \neq p_{q^{*}}$. Without loss of generality, it can be assumed that $p_{q}>p_{q^{*}} \geq c_{q}$. Then, object $q$ must be allocated to some buyer $b^{*}$ and $a_{b^{*} q}-p_{q}<a_{b^{*} q}-p_{q^{*}}=$ $a_{b^{*} q^{*}}-p_{q^{*}}$. This means that $b^{*} \notin B_{q}(p)$, which contradicts the competitivity of $p$. Then, $p_{q}=p_{q^{*}}$ and the proof is complete.

Proposition 3.3 The auction mechanism is manipulable by the buyers.

Proof We have to show that there is a competitive market for which straightforward behavior is not a dominant strategy for some buyer. Then, consider the market where $B=\left\{b_{1}, b_{2}, b_{3}, b_{o}\right\}, Q=\left\{1,2,3, q_{o}\right\}$ and $c=\{0,0,0,0\}$. The vector of values of the first three bidders are given by $(8,5,4,0),(2,4,4,0)$ and $(5,3,2,0)$, respectively. Without loss of generality, we can suppose that the starting price is $p(1)=(8,5,4,0)$. Bidder $b_{1}$ demands all four objects; bidder $b_{2}$ demands 3 and $q_{o}$. Bidder $b_{3}$ demands only $q_{o}$. Then, there is a minimal underdemanded set, $\{1,2\}$, whose set of demanders is $\left\{b_{1}\right\}$. The auctioneer lowers the prices of 1 and 2 by one unit. Thus, $p(2)=(7,4,4,0)$. Bidder $b_{1}$ demands 1 and 2 ; bidder $b_{2}$ demands 2,3 and $q_{o}$; bidder $b_{3}$ demands only $q_{o}$. There is again a minimal underdemanded set, $\{1,2,3\}$ whose set of demanders is $\left\{b_{1}, b_{2}\right\}$. Therefore, $p(3)=(6,3,3,0)$. At this step, $b_{1}$ demands 1 and 2 ; bidder $b_{2}$ demands 2 and 3 . If $b_{3}$ behaves straightforwardly, she will demand 2 and $q_{o}$, in which case the auction ends with the following outcome: $b_{1}$ receives object $1, b_{2}$ receives object 3 and $b_{3}$ receives object 2 . In this case, $b_{3}$ pays 3 for object 2 and gets a payoff of 0 . However, if $b_{3}$ behaves strategically and demands only $q_{o}$ at step $t=3$, then the minimal underdemanded set will be again $\{1,2,3\}$, so $p(4)=\{5,2,2,0\}$. The demands of bidders $b_{1}$ and $b_{2}$ do not change. We can see that bidder $b_{3}$ can get a payoff of 1 , if she demands $\left\{2, q_{o}\right\}$. Therefore, bidder $b_{3}$ can get a higher payoff than 0. (Indeed, there is a better manipulation for $b_{3}$ : she demands $q_{o}$, so the minimal underdemanded set will be again $\{1,2,3\}$, so $p(5)=\{4,1,1,0\}$. The demands of bidders $b_{1}$ and $b_{2}$ are the same as before. Then, bidder $b_{3}$ can get a payoff of 2 by demanding $\left.\left\{2, q_{o}\right\}\right)$.

We must remark that, in the proof of proposition 2 , for bidder $b_{3}$ to manipulate the mechanism, she does not need any information about the values of the other bidders. She only needs to observe which objects were demanded by each of her rivals. This contrasts sharply with the existent results in the literature, where a 
player needs to have a great deal of information to identify profitable strategic manipulations.

We are only interested in the manipulations by the buyers, since the reservation prices to the sellers are known at the beginning of the auction. However, sometimes the auctioneer can manipulate without changing his reservaton price. Suppose, for example, that she gets the information that buyer $b_{3}$, of the market described in the proof of proposition 2 has very low valuations for all items. After step $t=3$, all prices will decrease until bidder $b_{3}$ wants to stop the auction, by demanding some object different from $q_{o}$. Then, it is better for the auctioneer to stop the auction at step 3 and only sell objects 1 and 2 than wait until step $t=5$, for example, to sell all three objects. By doing this, she gets 9 instead of 6 ! She can do that by introducing at the beginning of the auction, a fictitious buyer who will demand object 3 at step $t=3$.

\section{Concluding Remarks}

Here we described a simultaneous descending auction to sell a set of indivisible items to a set of potential buyers. The auction is rather simple for the buyers: they only need to indicate their demands. The rules established by the auctioneer then determine the outcome.

The main characteristic of our auction mechanism is that the bids are dynamic and simultaneous. By use of dynamic bids, bidders can see how highly their opponents value each object and which objects they are looking for. As the auction approaches its end, each bidder is able to predict which object she will most probably get and to roughly calculate how much she will have to pay for it. Nonetheless, all objects are open for bidding simultaneously at each stage of the mechanism. Thereby, a bider is able to select whatever objects she wishes. Thus, efficiency is enhanced by dynamic and simultaneous bids.

Sequential auctions sell similar objects for quite different prices: objects sold at the beginning are usually able to reach better prices than those sold towards the end of the auction. (Ashenfelter, 1989); (McAfee and Vincent, 1993). Thus, a bidder can regret having purchased an object in an early auction, and not having waited for a better opportunity.

On the other hand, in the usual sealed bid auctions, bidders must bid blindly, unable to know how high they must bid to win a particular item. That means they run the risk of winning more than one object. As a direct consequence of such mechanism, budget-constrained bidders tend to bid low.

The theory developped in this paper shows that, in the simultaneous descend- 
ing auction here described, buyers do not win more than one object and similar objects are sold at the same prices. Moreover, the auction produces the same price vector and the same payoffs for the buyers, independently of the choices of the auctioneer.

Under the assumption that bidders construct their bids according to some matrix of indiretly revealed values, it can be proved that the dynamic auction is strategically equivalent to the following sealed-bid auction. Each buyer submits a sealed-bid listing her valuation of all the items. The auctioneer then allocates the objects in accordance with the maximum competitive equilibrium for the competitive market $\left(B, Q, a^{\prime}\right) .{ }^{8}$ Sotomayor (1999) demonstrates that, under the complete information approach, in the strategic game induced by this mechanism, every Nash equilibrium outcome is a competitive equilibrium under the true valuations. In this sense, the non-cooperative game can be regarded as a competitive process for achieving a cooperative outcome. ${ }^{9}$ Hence, efficiency is also guaranteed in the dynamic mechanism. ${ }^{10}$

In both auctions, it is assumed that a bidder's utility to an object is of the simple linear surplus type, already described. However, this is a very special case. We are sure that our dynamic mechanism allows the use of a greater variety of preferences for the bidders. The results demonstrated here suggest that for small enough bid decreases, our mechanism will produce prices approximating the maximum price equilibrium. Nonetheless, our results are given here only for the linear surplus case. The analysis for more general quotas remains a problem for future investigation and we hope that the model focused here is helpful in building understanding.

\footnotetext{
${ }^{8}$ In Demange (1982) and Leonard (1983)is presented a formula to determine the maximum competitive equilibrium price, as well as the minimum competitive equilibrium price.

${ }^{9}$ The idea of obtaining competitive allocations via a non-cooperative game was also explored in Wilson (1977). This author considers an exchange economy with indivisible goods, in which the trades are organized as a bidding game. Under several regularity assumptions (which do not apply to our model), it is shown that this game has an equilibrium which yields a core allocation of the corresponding cooperative game of exchange. The buyers are replicated and this core allocation is a competitive equilibrium in the limit.

${ }^{10}$ When the objects are identical, an efficient auction is one which puts goods into possession of the buyers who value them most. Among the many authors who have searched for efficient auctions, we can mention Dasgupta and Maskin (1998).
} 


\section{References}

Alkan, A. (1988). Auctionning several objects simultaneosly. mimeo., Bogazici University.

Alkan, A. (1992). Equilibrium in a Matching Market with General Preferences. The Macmillan Press Ltda. In: Majumdar, Mukul (ed.). Equilibrium and Dynamics: Essays in Honor of David Gale.

Alkan, A. and Gale, D. (1988). A constructive proof of non emptiness of the core of the mathcing game.

Ashenfelter, O. (1989). How auctions work for wine and art. Journal of Economic Perspectives, (3):23-36.

Crawford, V. P. and Knoer, E. M. (1981). Job matching with heterogeneous firms and workers. Econometrica, 49(2):437-450.

Dantzig, G. B. (1963). Linear Programming and Extensions. Princeton University Press.

Dasgupta, P. and Maskin, E. (1998). Efficient auctions. mimeo., Harvard University.

Demange, G. (1982). Strategyproofness in the assignment market game. Master's thesis, École Polytechnique, Laboratoire D'Econometrie, Paris.

Demange, G. and David, G. (1985). The strategy structure of two-sided matching markets. Econometrica, (53):873-883.

Demange, G., Gale, D., and Sotomayor, M. (1986). Multi-item auctions. Journal of Political Economy, 94(4):863-872.

Gale, D. (1960). The Theory of Linear Economic Models. McGraw-Hill, New York.

Gale, D. and Shapley, L. (1962). College admissions and the stability of marriage. American Mathematical Monthly, (69):9-15.

Hall, P. (1935). On representatives of subsets. J. London Math. Soc., (10):26-30.

Kaneko, M. (1982). The central assignment game and the assignment markets. Journal of Mathematics Economics, (10):205-232. 
Kaneko, M. and Yoshitsugu, Y. (1986). The existence and computation of competitive equilibria in markets with an indivisible commodity. Journal of Economic Theory, (38):118-136.

Leonard, H. B. (1983). Elicitation of honest preferences for the assignment of individuals to positions. Journal of Political Economy, pages 461-479.

McAfee, R. P. and Vincent, D. (1993). The declining price anomaly. Journal of Economic Theory, (60):191-212.

Quinze, M. (1984). Core and competitive equilibria with indivisibilities. International Journal of Game Theory, (13):41-60.

Roth, A. and Sotomayor, M. (1990). Two-Sided Matching. A Study in GameTheoretic Modeling and Analysis. Number 18 in Econometric Society Monograph Series. Cambridge University Press.

Shapley, L. and Shubik, M. (1972). The assignment game I: The core. International Journal of Game Theory, (1):111-130.

Sotomayor, M. (1986). On incentives in a two-sided matching market. preprint, Depart. of Mathematics, PUC/RJ.

Sotomayor, M. (1999). The lattice structure of the set of stable outcomes of the multiple partners assignment game. International Journal of Game Theory, (28):567-583.

Vickrey, W. (1961). Counterspeculation, auctions, and competitive sealed tenders. Journal of Finance, (16):8-37.

Wilson, R. (1977). Competitive exchange. Econometrica, 46(3):577-585. 